\title{
The influence of cardiovascular autonomic neuropathy on mortality in type 1 diabetic patients; 10-year follow-up
}

\author{
Silvie Lacigova, Jitka Brozova, Daniela Cechurova, Jitka Tomesova, Michal Krcma, Zdenek Rusavy
}

\begin{abstract}
Aim. The aim of our retrospective study was to answer the question if the presence of cardiovascular autonomic neuropathy (CAN) affects mortality in type 1 diabetic patients during a 10-year follow-up.

Methods. Patients with type 1 diabetes mellitus examined for CAN in 2003 were enrolled in this retrospective study. A total of 278 patients were included and divided into two groups according to the presence or absence of CAN (111 CAN+, 167 (AN-). The group characteristics and outcomes were compared at baseline and after ten years (in 2013).

Results. In the follow-up period, a total of 18 patients died; CAN+ $(14 / 111 ; 12.6 \%)$ and CAN- $(4 / 167 ; 2.4 \%)(P<0.001)$. At baseline, the CAN+ patients were older (47 vs. 33 years; $P<0.001$ ), had longer duration of diabetes ( 20 vs. 12 years; $P<0.05)$, had worse glycemic control assessed by HbA1c (73 vs. $68 \mathrm{mmol} / \mathrm{mol} ; P<0.05)$, higher systolic (130 vs. 120 $\mathrm{mmHg} ; P<0.001$ ) and diastolic ( 80 vs. $70 \mathrm{mmHg} ; P<0.01$ ) blood pressure and had more diabetic complications. In our analysis we found the strongest predictor of mortality to be the presence of CAN $(P<0.01)$ and the blood pressure value at baseline $(P<0.05)$. Other baseline characteristics, including the duration of diabetes, age and the presence of micro- and macrovascular complications were not significant. The statistical analysis was performed using logistic regression step-wise analysis.
\end{abstract}

Conclusions. During the 10-year follow-up, CAN+ patients had a 5-fold higher mortality rate than CAN-patients. The strongest predictor of mortality was the presence of CAN.

Key words: type 1 diabetic patients, cardiovascular autonomic neuropathy, chronic complications, morbidity, mortality

Received: February 26, 2015; Accepted with revision: December 2, 2015; Available online: January 5, 2016 http://dx.doi.org/10.5507/bp.2015.063

Department of Internal Medicine I, University Hospital in Pilsen and Faculty of Medicine in Pilsen, Charles University in Prague, Pilsen, Czech Republic

Corresponding author: Silvie Lacigova, e-mail: Lacigova@fnplzen.cz

\section{INTRODUCTION}

For a long time now, CAN has not been considered the Cinderella of diabetes complications ${ }^{1}$. Every year, numerous articles addressing this chronic complication come out; $99 \%$ of them stating that CAN significantly increases the morbidity and mortality of affected patients. This conclusion is based on pathophysiology, nevertheless, no clinician is comfortable with accepting the fact that $29 \%$ patients with CAN die within 10 years ${ }^{2}$ compared to $6 \%$ of patients without CAN. Our doubts regarding this information led us to organize this study. The outpatient clinic of the Diabetes Center in the University Hospital in Pilsen, Czech Republic follows about 600 patients with type 1 diabetes mellitus (DM1). Since 1998, examination of CAN is included in the regular screening for microvascular complications. The aim of this study was to compare the DM1 patients with and without CAN to answer the question if the presence of CAN affects the mortality of type 1 diabetic patients during a 10-year follow-up.

\section{METHODS}

Patients with DM1 examined for CAN in 2003 were enrolled in a retrospective study. The inclusion criteria were DM1 duration of more than one year before the examination for CAN, the absence of severe diabetes complications at the time of CAN examination (defined as the presence of end-stage renal disease (ESRD), history of major leg amputation, blindness), the absence of other severe diseases (malignancy, heart failure NYHA III-IV, alcoholism) and age between 18-70 years. In total, 278 patients were enrolled and divided into two groups according to the presence or absence of CAN (111 CAN+, 167 CAN-). The groups were compared at baseline and after ten years in 2013. The assessment of CAN was performed under strictly standardized conditions by a bedside on-line telemetric transmitter (VarCor PF7, DIMEA Group, Olomouc, Czech Republic). International recommendations were used for evaluation of the results ${ }^{3,4}$. Three tests from the Ewing battery were selected for CAN assessment: (i) Heart rate variability in response to deep breathing (parameter I/E), (ii) Laying-to-standing heart rate response (parameter RRmax/RRmin), (iii) Postural blood pressure change. The values of each patient were approximated to age according to international guidelines ${ }^{5-7}$. 
Patients having positive $\geq 2$ of the 3 tests were enrolled in the $\mathrm{CAN}+$ group and patients having positive $\leq 1$ test were included in the CAN-group.

The following variables were compared at baseline and after 10 years (median 9.72 years): glycemic control (HbAlc; mmol/mol), weight ( $\mathrm{kg})$, blood pressure (mean of two subsequent measured values; $\mathrm{mmHg}$ ), estimated Glomerular Filtration Rate (eGFR; mL/s) calculated as Modification of Diet in Renal Disease (MDRD). Albumin excretion rate from the collection of night urine (AER; ug/min; without AER < $20 \mathrm{ug} / \mathrm{min}$; with AER > $20 \mathrm{ug} /$ $\mathrm{min}$ ), retinopathy (ophthalmoscopy) and peripheral neuropathy (monofilaments; biothesiometer and Neuropad) were evaluated as present (1) or absent (0). Positivity of at least two out of three performed neuropathy tests was necessary for the diagnosis of neuropathy ${ }^{8}$. Diabetic foot (DF) was defined as a soft tissue ulceration or a bone affection under the level of the ankle associated with neuropathy and/or ischemia and/or amputation. The presence of cardiovascular disease was defined as the presence of one or more of the following: myocardial infarction, angina pectoris, a history of angioplasty or coronary bypass, transient ischemic attack, cerebrovascular event or the presence of peripheral vascular disease. The follow-up included the assessment of the presence of arterial hypertension on treatment, hyperlipoproteinemia and smoking.
The number of deaths and the cause of death were assessed. The data was obtained from the Diabetes Center of the University Hospital in Pilsen registry and from the Department of Forensic Medicine of the Medical Faculty in Pilsen, Charles University in Prague.

Data were tested for normality and parametric and non-parametric comparisons were used as appropriate. Median and interquartile range were used for descriptive analysis. Median differences between baseline and 10-year follow-up were compared using Wilcoxon unpaired test, categorical variables were analyzed with Chi-square test $\left(\chi^{2}\right)$. Stepwise logistic regression analysis was used for the assessment of the risk factors for mortality.

\section{RESULTS}

Severe CAN was diagnosed in 111 (39.9\%) of the enrolled 278 DM1 patients that were examined for the presence of CAN in 2002-2004 and fulfilled the inclusion criteria. The baseline characteristics and group comparison are shown in Table 1 and 2.

At baseline, the patients in CAN+ were older ( 47 vs. 33 years; $P<0.001)$, had longer duration of diabetes ( 20 vs. 12 years; $P<0.05$ ), worse glycemic control (HbA1c) ( 73 vs. $68 \mathrm{mmol} / \mathrm{mol} ; P<0.05$ ), higher systolic

Table 1. Baseline characteristic of group CAN+ and CAN-.

\begin{tabular}{lccc}
\hline & $\begin{array}{c}\text { CAN+ } \\
(\mathrm{n}=111)\end{array}$ & $\begin{array}{c}\text { CAN- } \\
(\mathrm{n}=167)\end{array}$ & $\begin{array}{c}P \\
\text { (Wilcoxon unpaired test) }\end{array}$ \\
\hline Age (years) & $47(36 ; 56)$ & $33(26 ; 42)$ & $P<0.001$ \\
Duration of DM (years) & $20(13 ; 27)$ & $12(7 ; 19)$ & $P<0.05$ \\
Age of DM manifestation (years) & $25(17 ; 34)$ & $20(14 ; 30)$ & $P<0.05$ \\
Weight (kg) & $73(65 ; 81)$ & $73(65 ; 82)$ & NS \\
Systolic blood pressure (mmHg) & $130(120 ; 135)$ & $120(110 ; 130)$ & $P<0.001$ \\
Diastolic blood pressure (mmHg) & $80(70 ; 80)$ & $70(70 ; 80)$ & $P<0.01$ \\
HbAlc (mmol/mol) & $73(63 ; 83)$ & $68(59 ; 80)$ & $P$ \\
\hline
\end{tabular}

Results are given as median (interquartile range).

$\mathrm{CAN}+/ \mathrm{CAN}$ - the group with/without cardiovascular autonomic neuropathy; HbAlc, glycosylated hemoglobin; NS, not significant.

Table 2. Morbidity of group CAN+ and group CAN- at baseline.

\begin{tabular}{lccc}
\hline & $\begin{array}{c}\text { CAN }+ \\
(\mathrm{n}=111)\end{array}$ & $\begin{array}{c}\text { CAN- } \\
(\mathrm{n}=167)\end{array}$ & $\begin{array}{c}P \\
\text { (Wilcoxon unpaired test) }\end{array}$ \\
\hline Retinopathy & $75(67.6 \%)$ & $36(21.6 \%)$ & $P<0.001$ \\
AER & $46(41.4 \%)$ & $19(11.4 \%)$ & $P<0.001$ \\
eGFR (mL/s) & $1.16(0.95 ; 1.39)$ & $1.35(1.21 ; 1.49)$ & $P<0.001$ \\
Neuropathy & $66(59.5 \%)$ & $31(18.6 \%)$ & $P<0.001$ \\
Diabetic foot & $7(6.3 \%)$ & $0(0 \%)$ & $P<0.01$ \\
Cardiovascular disease & $7(6.3 \%)$ & $11(0.6 \%)$ & $P<0.01$ \\
Treatment of AH & $36(32.4 \%)$ & $28(16.8 \%)$ & $P<0.001$ \\
Treatment of HLP & $33(29.7 \%)$ & $36(21.6 \%)$ & $P<0.05$ \\
Smoking & $21(19 \%)$ & NS \\
\hline
\end{tabular}

Results are given as number of cases (\%) or as median (interquartil range).

$\mathrm{CAN}+/ \mathrm{CAN}$-, the group with/without cardiovascular autonomic neuropathy; AER, albumin excretion rate; eGFR, estimated glomerular filtration rate; $\mathrm{AH}$, arterial hypertension; HLP, hyperlipoproteinemia; $\chi 2$, Chi-square test; NS, not signifiant. 
(130 vs. $120 \mathrm{mmHg} ; P<0.001)$ and diastolic ( 80 vs. 70 mmHg; $P<0.01)$ blood pressure and lower eGFR (1.16 vs. $1.35 \mathrm{~mL} / \mathrm{s} ; P<0.001)$. The presence of retinopathy (67.6 vs. $21.6 \% ; P<0.001)$, peripheral neuropathy $(59.5$ vs. $18.6 \% ; P<0.001)$, DF (6.3 vs. $0 \% ; P<0.01)$ and AER (41.4 vs. $11.4 \% ; P<0.001$ ) was also more frequent in the CAN+ group at baseline. Higher presence of CVD ( 6.3 vs. $0.6 \% ; P<0.01)$, hypertension on treatment $(32.4$ vs. $6.6 \% ; P<0.001)$ and hyperlipoproteinemia $(29.7$ vs. $16.8 \% ; P<0.05)$ in the CAN+ group was also observed. The proportion of smokers ( 19 vs. $21.6 \%$ ) was similar in both groups.

In the follow-up period, a total of 18 patients died $(18 / 278 ; 6.5 \%) ; 14$ from the CAN+ group $(14 / 111 ; 12.6 \%)$ and 4 from the CAN-group $(4 / 167 ; 2.4 \%)(P<0.001)$ (Table 3$)$. The main predictors of mortality were the presence of CAN $(P<0.01)$ and systolic blood pressure at baseline $(P<0.05)$. Duration of diabetes at baseline, age and the presence of micro- and macrovascular complications were not significantly associated with the mortality rate (Table 4).

Analysis of the cause of death showed that 8 patients died of diabetes complications, 3 patients of CVD and 7 patients died of other causes (Table 5).

\section{DISCUSSION}

The prevalence of CAN in our group of patients was $39.9 \%(111 / 278)$. This number is comparable with our previous results ${ }^{9}$, as well as with the results published by other authors. The prevalence of CAN is usually reported to be between 20 and $65 \%$ (ref. ${ }^{10}$ ). However, there is wide variability, from $2.5 \%$, in patients with short duration of diabetes ${ }^{11}$ up to $90 \%$ in patients waiting for pancreas transplant $^{12}$. CAN is often asymptomatic for a long time, as seen in our study, and it is rarely included in the screening of chronic complications. This can explain the differences in the prevalence observed amongst the studies. In addition, the rate of CAN diagnoses depends on the type and number of cardiovascular tests used (e.g. Ewing battery, Spectral analysis of heart rate variability). To date, there is no consensus regarding the optimal screening for CAN. If the evaluated group is large, even one test can be sufficient to diagnose CAN, as seen in the EURODIAB study $^{13}$. In a consensus published in 2011 by a working group lead by Spallone, at least two abnormal cardio-vagal tests and the presence of orthostatic hypotension are recommended for the identification of severe or advanced CAN (ref. ${ }^{10}$ ). In our study, the selection of the tests was the same (I/E, RRmax/RRmin, orthostatic hypotension test). According to May and Arildsen, each test from the Ewing battery holds a significant predictive value for the assessment of mortality ${ }^{14}$.

In agreement with the studies referenced below, we showed that the patients in the CAN+ were older and had a longer duration of diabetes. The factor of time in the relation to the presence of CAN is logical. The reactivity of the autonomic nervous system naturally decreases with age, therefore the results of individual tests must be approximated to age s-7 $^{5}$ There are only a few studies demonstrating the presence of CAN early after the manifestation of DM (ref. ${ }^{15}$ ). Poor diabetes control itself is considered to be an another risk factor for the development of CAN, especially in DM1 ( ref. $^{10}$ ). There is no doubt that hyperglycemia drives the pathophysiological processes leading to virtually all chronic complications of diabetes. Also for CAN, the effect of good ${ }^{11}$ and especially early glycemic control has been demonstrated. In the DCCT study, the conventionally treated group showed an increase in CAN as high as $35 \%$, compared to the intensively-treated group ${ }^{16}$. The effect of good glycemic control on the sympathetic innervation of the myocardium was demonstrated by Ziegler in his 4-year prospective study. Using metaiodobenzylguanidine scintigraphy, he was able to prove that the autonomic innervation was worse in the

Table 3. Mortality in group CAN+ and CAN- during 10 years follow up.

\begin{tabular}{lccc}
\hline & CAN $+(\mathrm{n}=111)$ & CAN- $(\mathrm{n}=167)$ & $P$ \\
\hline Mortality & $14(12.6 \%)$ & $4(2.4 \%)$ & $P<0.001$ \\
\hline
\end{tabular}

Results are given as number of cases (\%)

$\mathrm{CAN}+/ \mathrm{CAN}-$, the group with/without cardiovascular autonomic neuropathy; $\chi 2$, Chi-square test.

Table 4. Predictors of mortality.

\begin{tabular}{lcc}
\hline & Odds ratio & $P$ \\
\hline Age (basal) & - & NS \\
Duration of DM & - & NS \\
Weight (basal) & - & NS \\
HbA1c (mmol/mol) & - & NS \\
Systolic BP (basal) & $0.04-1.04$ & 0.05 \\
Diastolic BP (basal) & - & 0.05 \\
Presence of CAN & $1.48-4.38$ & 0.01 \\
\hline
\end{tabular}

Multivariate stepwise logistic regression. 
group of poorly controlled DM1 patients in comparison to the well-controlled group ${ }^{17}$. Even stronger evidence of positive effect of normoglycaemia is provided in a recently published study ${ }^{18}$.

Our group of $\mathrm{CAN}+$ patients had a higher overall presence of microvascular complications at baseline. The mutual relationship of CAN and these complications has been assessed in a number of studies ${ }^{19-20}$. In our previous study, we were able to demonstrate that the presence of CAN can be predicted based on the mutual relationship with other chronic complications. A patient with concurrent peripheral neuropathy, hypertension and microalbuminuria has as well a high probability of having $\mathrm{CAN}^{9}$. In our study, these complications were present concomitantly as well. Peripheral neuropathy was present in $59.5 \%$ of patients with CAN and only in $18.6 \%$ of patients without CAN (Table 2). CVD, hypertension on treatment and hyperlipoproteinemia were too found more frequently in the CAN+ group already at baseline. These risks, however, are more commonly found in patients with type 2 diabetes mellitus ${ }^{10}$. In one issue of EURODIAB Prospective Complications Study, Witte suggested that the risk factors of CAN could be of equal importance as the classical cardiovascular risk factors ${ }^{21}$. Similar results were presented in another EURODIAB study led by Kempler et al. ${ }^{13}$. In a group of 3007 DM1 patients, he was able to demonstrate

Table 5. Analysis of mortality in the group $\mathrm{CAN}+$ and $\mathrm{CAN}$ - during 10 years follow up.

\begin{tabular}{|c|c|c|c|c|}
\hline & & Causes of death & $\begin{array}{l}\text { Age in time } \\
\text { of death }\end{array}$ & $\begin{array}{l}\text { Duration of } \\
\text { diabetes }\end{array}$ \\
\hline \multirow[t]{14}{*}{$\mathrm{CAN}+$} & \multirow{4}{*}{$\begin{array}{l}\text { Cardiovascular mortality } \\
(\mathrm{n}=4)\end{array}$} & Chronic heart failure & 71 & 45 \\
\hline & & Myocardial infarct & 49 & 22 \\
\hline & & Ischemic stroke & 62 & 12 \\
\hline & & Ischemic stroke & 75 & 45 \\
\hline & \multirow{7}{*}{$\begin{array}{l}\text { DM-related mortality } \\
(\mathrm{n}=7)\end{array}$} & ESRD & 62 & 39 \\
\hline & & ESRD & 58 & 41 \\
\hline & & ESRD & 68 & 39 \\
\hline & & ESRD & 42 & 23 \\
\hline & & Hypoglycaemia & 49 & 12 \\
\hline & & Hypoglycaemia & 70 & 40 \\
\hline & & Hypoglycaemia & 28 & 26 \\
\hline & \multirow{3}{*}{$\begin{array}{l}\text { Other reasons of mortality } \\
(\mathrm{n}=3)\end{array}$} & Tentamen suicidii & 68 & 26 \\
\hline & & Pneumonia & 68 & 29 \\
\hline & & Breast carcinoma & 51 & 22 \\
\hline \multirow[t]{5}{*}{ CAN - } & $\begin{array}{l}\text { Cardiovascular mortality } \\
(\mathrm{n}=0)\end{array}$ & & & \\
\hline & \multirow{4}{*}{$\begin{array}{l}\text { DM-related mortality } \\
(n=2) \\
\text { Other reasons of mortality } \\
(n=2)\end{array}$} & Diabetic ketoacidosis & 28 & 11 \\
\hline & & ESRD & 25 & 14 \\
\hline & & Urinary bladder carcinoma & 57 & 21 \\
\hline & & Intracerebral hemorrhage & 39 & 22 \\
\hline
\end{tabular}

Table 6. Mortality of diabetic patients with/without CAN.

\begin{tabular}{|c|c|c|c|c|}
\hline Studies & Follow-up (years) & $\begin{array}{c}\text { Mortality CAN+ } \\
\text { n (\%) }\end{array}$ & $\begin{array}{c}\text { Mortality CAN- } \\
\mathrm{n}(\%)\end{array}$ & $P$ \\
\hline Ewing, 1980 & 5 & $21 / 40(53)$ & $5 / 33(15)$ & $P<0.05$ \\
\hline $\begin{array}{l}\text { Hasslacher and } \\
\text { Bassler, } 1983\end{array}$ & 5 & $3 / 16(19)$ & $3 / 42 \quad(7)$ & NS \\
\hline Navarro, $1990^{28}$ & 3.3 & $41 / 175(23)$ & $2 / 57 \quad(4)$ & $P<0.05$ \\
\hline Sampson, 1990 & 10 & $18 / 49$ & 4/38 (11) & $P<0.05$ \\
\hline O’Bien, 199129 & 5 & $23 / 84(27)$ & $21 / 422 \quad(5)$ & $P<0.05$ \\
\hline Ewing, 1991 & 3 & $10 / 32(31)$ & $3 / 39 \quad(8)$ & $P<0.05$ \\
\hline Jermendy, 1991 & 5 & $12 / 30(40)$ & $1 / 23$ & $P<0.05$ \\
\hline Rathmann, $1993^{27}$ & 8 & $8 / 35(23)$ & $1 / 35 \quad(3)$ & $P<0.05$ \\
\hline Luft, 1993 & 8 & $7 / 34(21)$ & $1 / 19 \quad(5)$ & NS \\
\hline Total & 5.8 & $143 / 495$ (29) & $41 / 708 \quad(6)$ & \\
\hline
\end{tabular}

Modification from Ziegler $^{2}$

$\mathrm{CAN}+/ \mathrm{CAN}-$, the group with/without cardiovascular autonomic neuropathy; NS, not significant. 
that the presence of CAN was associated with age, diabetes duration, glycemic control, blood pressure, lower level of HDL cholesterol, total cholesterol/HDL cholesterol ratio, triglycerides, the presence of retinopathy and albuminuria, and smoking. In our study however, the number of smokers in both groups was similar.

In spite of the decreasing overall mortality rate of DM1 patients and the markedly extending period from diabetes onset to death, the prognosis of the patients with DM1 is still worse than in the non-diabetic population. A recently published study suggests a strong relationship between poor glycemic control and excess of both total and cardiovascular mortality in patients with DM1 $\left(\right.$ ref. $\left.^{22}\right)$

A British study has shown the annual mortality in DM1 patients to be $8 / 1000$, whereas in the non-diabetic population only $2.4 / 1000$. The increased mortality in DM1 patients has been observed in all age groups. In gender analysis, the difference turned out to be even more pronounced in women ${ }^{23}$. The number of deaths and the cause of death in DM1 patients may be additionally influenced by the factor of time - most importantly by the duration of diabetes and the calendar year of diabetes onset $^{24}$. A Japanese study assessing the causes of death in childhood-onset DM1 patients found that CVDs were the main causes of the death of patients with duration of diabetes longer than 20 years. Therefore is necessary to start monitoring the cardiovascular risk factors early ${ }^{25}$, as the age of onset (before/after puberty) may be a risk factor for the development of complications. An Italian study confirmed that the mortality of DM1 patients is twice as high as the general Italian population of the same age, and those with DM onset after puberty are at higher risk of mortality ${ }^{26}$. Characterizing the whole group of patients followed in our study, the median age at baseline (years 2002-2004) was 37.5 years, with the mean diabetes duration of 15 years (onset in 1989) and manifestation after puberty in 22.5 years (Table 1 ). The overall group mortality rate was $6.5 / 1000 /$ year.

If we assess the mortality rate in relation to the presence or absence of CAN, we can look at the most cited works in this area, Ziegler et al. ${ }^{2}$ and Rathmann et al. ${ }^{27}$ and conclude that there is an increased mortality of patients with CAN. Ziegler et al. performed a meta-analysis of 9 studies showing that $29 \%$ of diabetics with CAN compared to $6 \%$ of diabetics without CAN die within 10 years (Table 6). However, only one study followed the patients for 10 years, with two other studies having an 8 year follow-up, four studies having only 5 years of followup data, and in Ewing's study following the patients for only 3 years (mean length of follow-up of all studies 5.8 years). All the studies differed in the number and type of the Ewing battery tests used (in four studies, only 1-2 tests were used and one study used all five tests, with three studies adding the QTc parameter to the testing battery). Overall, in all 9 studies there were 495 patients with CAN and 708 patients without CAN and $29 \%$ of patients with CAN (143) compared with 6\% (41) without CAN died. In two studies the difference in mortality between the two studied groups was statistically insignificant, in seven it reached statistical significance. Not all patients had DM1. Only two studies followed a larger group of patients (232 and 506), however the follow-up time was relatively short, only 3.3 and 5 years, respectively ${ }^{28,29}$. The remaining seven studies had small sample sizes, ranging from 53 to 87 patients. One of the 9 studies mentioned is that of Rathmann et al. ${ }^{27}$, where at the beginning of this study, more than 1000 patients were examined and only 35 patients with CAN and 35 without CAN were selected for the 8-year follow-up. Within the eight years, $23 \%$ (8) of patients from the CAN group compared to $3 \%$ (1) without CAN died. This study included both DM1 and DM2 patients.

In our study groups, only DM1 patients were included, the length of follow-up was 10 years (mean 9.72) and three tests were used for diagnostics of CAN. The presence of CAN and the systolic blood pressure value at baseline were identified as the strongest predictors of mortality. The glycemic control at baseline was approaching significance. Baseline diabetes duration, age as well as other variables (the presence of micro- and macrovascular complications) were not significant (in a logistic regression, step-wise analysis). In our study, the percentage of patient death was about a half in both groups $(12.4 \%$ vs. $2.4 \%$ ) compared to Ziegler's meta-analysis (29\% vs. $6 \%$ ), but the relative difference in mortality between the group with and without CAN was comparable. In our study, 5.3 times more patients from the CAN+ group died compared to CAN-group. In Ziegler's meta-analysis, the difference in mortality was 4.8 -fold. The greater number of deceased patients in Ziegler's meta-analysis could be explained by the design of the individual studies, where older patients with type 2 diabetes were also enrolled. Furthermore, the fact that the studies included in the meta-analysis were performed more than 20 years ago could play an important role. General care of a patient with diabetes mellitus and CVD has changed dramatically over the 20 years. The presence of CAN was classified as a strong predictor of mortality even by the EURODIAB study authors. Microvascular complications, such as macroalbuminuria and peripheral and autonomic neuropathy, were considered strong markers of future mortality risk and exceed the traditional cardiovascular risk factors in their importance ${ }^{30}$. The negative effect of CAN on inpatient and long-term mortality after a myocardial infarction was confirmed in a study from Montenegro. Patients with DM and CAN had worse results in all observed variables than DM patients without CAN, while the results of DM patients were generally worse than of non-DM patients ${ }^{31}$.

It is difficult to prove that CAN responsible for a death. Malignant arrhythmia is mentioned most frequently, especially if elongation of the QTc interval was documented. A myocardial contractility disorder leading to diastolic dysfunction has other causes. A further possibility is a central respiratory disorder and „dead in bed syndrome" attributed to malignant arrhythmia induced by increased sympathetic nervous system tone during hypoglycemia $^{32}$. The causes of death in our group are presented 
as individual cases in Table 5. Even though diabetic nephropathy in the ESRD stage is not anymore the leading cause of death, nephropathy is a factor that potentiates the very cardiovascular mortality of this group up to tenfold $^{33}$. Most studies conclude that if DM1 patients die within 10 years of diabetes duration, the death is usually caused by acute complications (hypoglycemia, diabetic ketoacidosis). If the DM1 duration is about 20 years, ESRD has a share, and in diabetes lasting for longer than 20 years, the leading cause of death is CVD. Even in our small study, the causes of death follow a similar pattern ${ }^{34}$.

\section{Study limitations and strengths}

The strength of the study is the duration of follow-up of a large group of DM1 patients. The depth of source data collection, including information regarding death are also advantages. The main limitations may be the retrospective design and the fact that it is a single-center study. Use of modified tests of heart rate variability spectral analysis ${ }^{35}$ might contribute to facilitation and spread of CAN assessment.

\section{CONCLUSIONS}

Despite the original objective of the study to rebut the pessimistic prediction of CAN prognosis, our retrospective study confirmed the seriousness of CAN. Over the 10-year follow-up, the mortality rate was 5 times higher in the group of DM 1 patients with CAN compared to patients without CAN. Although there were already baseline differences between $\mathrm{CAN}+$ and $\mathrm{CAN}$ - patients in age and morbidity, the presence of CAN was statistically the strongest predictor of mortality.

Acknowledgement: This study was supported by the Charles University Research Fund (project number P36). Author contributions: SL: data selection, manuscript writing; JB, JT, MK: statistical analysis and tablets; DC, ZR: text revision.

Conflict of interest statement: None declared.

\section{REFERENCES}

1. Vinik Al, Erbas T. Diabetic autonomic neuropathy. Handb Clin Neurol 2013;117:279-94.

2. Ziegler D. Diabetic cardiovascular autonomic neuropathy: prognosis, diagnosis and treatment. Diabetes/Metabolism Reviews 1994;10(4):339-83.

3. Standards of Mediacal Care in Diabetes 2015. American Diabetes Association. Diabetes Care 2015;38:(S1):S58-S66.

4. Metelka R. Heart rate variability - current diagnosis of the cardiac autonomic neuropathy. A review. Biomed Pap Med Fac Univ Palacky Olomouc Czech Repub 2014;158(3):327-38.

5. Ewing DJ, Martyn CN, Young RJ, Clarke BF. The value of cardiovascular autonomic function tests: 10 years experience in diabetes. Diabetes Care 1985;8(5)491-8.

6. Wieling W, van Brederode JF, de Rijk LG, Borst C, Dunning AJ. Reflex control of heart rate in normal subjects in relation to age: a data base for cardiac vagal neuropathy. Diabetologia 1982;22(3):163-6.

7. Gautschy B, Weidmann P, Gnadinger MP. Autonomic function tests as related to age and gender in normal man. Klin Wochenschr 1986;64(11):499-505.
8. American Diabetes Association. Clinical Practice Recommendations. Diabetes Care 2014; 37(S1):S46.

9. Lacigova S, Safranek P, Cechurova D, Krcma M, Visek J, Jankovec Z, Zourek M, Haladova I, Rusavy Z . Could we predict asymptomatic cardiovascular autonomic neuropathy in type 1 diabetic patients attending out-patients clinics? Wiener Klin Wochenschrift 2007;119(910):303-8.

10. Spallone V, Ziegler D, Freeman R, Bernardi L, Frontoni S, Pop-Busui R, Stevens M, Kempler P, Hilsted J, Tesfaye S, Low P, Valensi P; Toronto Consensus Panel on Diabetic Neuropathy. Cardiovascular autonomic neuropathy in diabetes: clinical impact, assessment, diagnosis, and management. Diabetes Metab Res Rev 2011;27(7):639-53.

11. The effect of intensive diabetes therapy on measures of autonomic nervous system function in The Diabetes Control and Complications Trial (DCCT). (No authors listed). Diabetologia 1998;41(4):416-23.

12. Kennedy WR, Navarro $X$, Sutherland DE. Neuropathy profile of diabetic patients in a pancreas transplantation program. Neurology 1995;45(4):773-80.

13. Kempler P, Tesfaye S, Chaturvedi N, Stevens LK, Webb DJ, Eaton S, Kerényi Z, Tamás G, Ward JD, Fuller JH. Autonomic neuropathy is associated with increased cardiovascular risk factors: the EURODIAB IDDM Complications Study Diabet Med 2002;19(11):900-9.

14. May $\mathrm{O}$, Arildsen $\mathrm{H}$. Long-term predictive power of simple function tests for cardiovascular autonomic neuropathy in diabetes: a population-based study. Acta Diabetol 2011;48(4):311-6.

15. Ziegler D, Dannehl K, Volksw D, Mühlen H, Spüler M, Gries FA. Prevalence of cardiovascular autonomic dysfunction assessed by spectral analysis and standard tests of heart-rate variation in newly diagnosed IDDM patients. Diabetes Care 1992;15(7):908-11.

16. Pop-Busui R, Low PA, Waberski BH, Martin CL, Albers JW, Feldman EL, Sommer C, Cleary PA, Lachin JM, Herman WH. Effects of prior intensive insulin therapy on cardiac autonomic nervous system function in type 1 diabetes mellitus: the Diabetes Control and Complications Trial/Epidemiology of Diabetes Interventions and Complications study (DCCT/EDIC). Circulation 2009;119(22):2886-93.

17. Ziegler D, Weise F, Langen KJ, Piolot R, Boy $C$, Hübinger A, MüllerGärtner HW, Gries FA. Effect of glycaemic control on myocardial sympathetic innervation assessed by[1231] metaiodobenzylguanidine scintigraphy: a 4-year prospective study in IDDM patients. Diabetologia 1998;41(4):443-51.

18. Ziegler D, Behler M, Schroers-Teuber M, Roden M. Near-normoglycaemia and development of neuropathy: a 24-years prospective study from diagnosis of type 1 diabetes. BMJ Open 2015;5:e006559.

19. Weinrauch LA, Kennedy FP, Gleason RE, Keough J, D'Elia JA. Relationship between autonomic function and progression of renal disease in diabetic proteinuria: clinical correlations and implications for blood pressure control. Am J Hypertens 1998;11(3):302-8.

20. Astrup AS, Tarnow L, Rossing P, Hansen BV, Hilsted J, Parving HH. Cardiac autonomic neuropathy predicts cardiovascular morbidity and mortality in type 1 diabetic patients with diabetic nephropathy. Diabetes Care 2006;29(2):334-9.

21. Witte DR, Tesfaye S, Chaturvedi N, Eaton SEM, Kempler P, Fuller JH. EURODIAB Prospective Complications Study Group. Risk factors for cardiac autonomic neuropathy in type 1 diabetes mellitus. Diabetologia 2005;48(1):164-71.

22. Lind $M$, Svensson $A M$, Kosiborod M, Gudbjörnsdottir S, Pivodic A, Wedel H, Dahlqvist S, Clements M, Rosengren A. Glycemic control and excess mortality in type 1 diabetes. $\mathrm{N}$ Engl J Med 2014;371(21):1972-82.

23. Soedamah-Muthu SS, Fuller JH, Mulnier HE, Raleigh VS, Lawrenson RA, Colhoun HM. All-cause mortality rates in patients with type 1 diabetes mellitus compared with a non-diabetic population from the UK general practice research database, 1992-1999. Diabetologia 2006;49(4):660-6.

24. Pambianco G, Costacou T, Ellis D, Becker DJ, Klein D, Orchard TJ. The 30-Year Natural History of Type 1 Diabetes Complications. The Pittsburgh Epidemiology of Diabetes Complications Study Experience. Diabetes 2006;55(5):1463-9.

25. Morimoto A, Onda Y, Nishimura R, Sano H, Utsunomiya K, Tajima N. Cause-specific mortality trends in a nationwide populationbased cohort of childhood-onset type 1 diabetes in Japan during 35 years of follow-up: the DERI Mortality Study. Diabetologia 2013; 56(10):2171-5.

26. Bruno G, Cerutti F, Merletti F, Novelli G, Panero F, Zucco C, CavalloPerin P; Piedmont Study Group for Diabetes Epidemiology. Short- 
term mortality risk in children and young adults with type 1 diabetes: the population-based registry of the province of Turin, Italy. Nutr Metab Cardiovasc Dis 2009;19(5):340-4.

27. Rathmann W, Ziegler D, Jahnke M, Haastert B, Gries FA. Mortality in diabetic patients with cardiovascular autonomic neuropathy. Diabet Med 1993;10(9):820-4.

28. Navarro X, Kennedy WR, Loewenson RB, Sutherland DER. Influence of pankreas transplantation on cardiorespiratory reflexes, nerve conduction, and mortality in diabetes. Diabetes 1990;39(7):802-6.

29. O'Brien IA, McFadden JP, Corral RJM. The influence of autonomic neuropathy on mortality in insulin-dependent diabetes. QJMed 1991;79(290):495-502.

30. Soedamah-Muthu SS, Chaturvedi N, Witte DR, Stevens LK, Porta $\mathrm{M}$, Fuller JH. Relationship between risk factors and mortality in type 1 diabetic patients in Europe. The EURODIAB Prospective Complications Study. Diabetes Care 2008;31(7):1360-6.

31. Vujosevic S, Zamaklar M, Belada N, Stamatovic S. Mortality after acute myocardial infarction: significance of cardiovascular diabetic autonomic neuropathy. Med Arh 2012;66(5):296-9.

32. Bell DS. Dead in bed syndrome - a hypothesis. Diabetes Obes Metab 2006;8(3):261-3.

33. Mäkinen VP, Forsblom C, Thorn LM, Wadén J, Gordin D, Heikkilä $\mathrm{O}$, Hietala K, Kyllönen L, Kytö J, Rosengård-Bärlund $\mathrm{M}$, Saraheimo M, Tolonen N, Parkkonen M, Kaski K, Ala-Korpela M, Groop PH; FinnDiane Study Group. Metabolic phenotypes, vascular complications, and premature deaths in a population of 4,197 patients with type 1 diabetes. Diabetes 2008;57(9):2480-7.

34. Maahs DM, Rewers B. Mortality and renal disease in type 1 diabetes mellitus-progress made, more to be done. J Clin Endocrinol Metab 2006;91(10):3757-9.

35. Howorka K, Pumprla J, Jirkovska A, Lacigova S, Nolan J. Modified orthostatic load for spectral analysis of short-term heart rate variability improves the sensitivity of autonomic dysfunction assessment. J Diabetes Complications 2010;24(1):48-54. 